Mots. Les langages du politique

Guerres et paix. Débats, combats, polémiques

\title{
Dialoguer au cœur du conflit? Lettres ouvertes franco-allemandes, 1870/1914
}

Keeping dialogue in the heat of the conflict ? Franco-german open letters, 1870/1914

¿ Dialogar en pleno conflicto? El caso de las cartas abiertas franco-alemanas, 1870-1914

Ruth Amossy

\section{(2) OpenEdition}

\section{Journals}

Édition électronique

URL : https://journals.openedition.org/mots/2013

DOI : 10.4000/mots.2013

ISSN : 1960-6001

Éditeur

ENS Éditions

Édition imprimée

Date de publication : 1 novembre 2004

Pagination : 25-39

ISBN : 2-84788-064-X

ISSN : 0243-6450

\section{Référence électronique}

Ruth Amossy, «Dialoguer au cœur du conflit ? Lettres ouvertes franco-allemandes, 1870/1914 »,

Mots. Les langages du politique [En ligne], 76 | 2004, mis en ligne le 21 avril 2008, consulté le 23 avril 2022. URL : http://journals.openedition.org/mots/2013 ; DOI : https://doi.org/10.4000/mots.2013 


\section{Dialoguer au cœur du conflit ? Lettres ouvertes franco-allemandes, 1870/1914}

Lettres à un ami allemand: le titre proposé par Albert Camus ne déparerait pas la correspondance qui voit le jour dans la presse française au moment de la guerre franco-prussienne de 1870, puis de la Grande Guerre en 1914. Car ces lettres ouvertes s'adressent toutes à un membre éminent de la nation adverse, et cela au plus fort des combats. Dans les cas, désormais célèbres, d'Ernest Renan ou de Romain Rolland, on a affaire à un véritable échange de correspondance puisque le savant David -Friedrich Strauss, comme l'écrivain Gerhart Hauptmann, font paraitre dans les journaux de leur pays leurs propres missives à l'intention de leurs collègues français.

On peut se demander quels peuvent être les objectifs et les enjeux d'une interaction épistolaire publique entre individus appartenant à deux nations en guerre. Dans le cadre de la rhétorique entendue comme art de persuader, ce qui fait problème, c'est la pertinence d'un discours adressé à l'autre au moment d'un déferlement de violence que le logos a échoué à endiguer. On sait que l'argumentation est censée offrir un substitut à la force brute (Perelman et Olbrechts-Tyteca, 1970). Est-il possible de préserver ses droits et ses pouvoirs en des circonstances où tout discours risque de paraitre futile ? Rappelons la formule cinglante qui ouvre en 1915 le pamphlet d'Henri Massis, Romain Rolland contre la France : «M. Rolland parle et la France se bat».

Si cette question relève autant de l'Histoire que des théories de l'argumentation, c'est que les possibilités de dialogue entre ennemis nationaux dépendent nécessairement d'une «culture de guerre» déterminée (Audoin-Rouzeau et Becker (éd.), 1994; Rioux et Sirinelli (éd.), 1997; Prost et Winter, 2004). La lettre ouverte dévoile la marge de manœuvre que chaque conflit assigne au partage de la parole. Ce faisant, le débat qui se développe dans la presse dévoile les modifications que subit le rapport au logos et à la violence dans la relation FranceAllemagne de 1870 à 1914. C'est dans ce sens que les modalités argumentatives

1. Université de Tel-Aviv - amossy@attglobal.net 
du dialogue sur la guerre et la paix sont une pièce à verser au dossier de l'Histoire : l'argumentation dans le discours (Amossy, 2000) rencontre ici l'Histoire culturelle de la Grande Guerre.

C'est en un premier temps sur le genre de discours choisi, en l'occurrence la lettre publique, et plus particulièrement la lettre ouverte, qu'il faut s'interroger. Le terme en vient à désigner dans le dernier tiers du $19^{\mathrm{e}}$ siècle un «Article de journal ou opuscule, généralement de caractère polémique, rédigé sous forme de lettre» $(T L F, 1983)^{2}$. En d'autres termes, la forme de l'épistolaire est exploitée pour exprimer un désaccord ou intervenir dans une polémique concernant une affaire publique. Encore faut-il distinguer «les missives adressées à un interlocuteur-destinataire collectif de la lettre ouverte» à proprement parler. La première, note Jean-Michel Adam, se contente de démultiplier le destinataire; la seconde «joue sur la désignation-prétexte ou postiche d'un interlocuteur unique» (Adam, 1998, p. 50); elle s'adresse à un correspondant privilégié tout en se destinant, à travers lui, à un vaste public. C'est le cas de certaines lettres ouvertes qui se distinguent en cela des manifestes et écrits collectifs qui abondent durant la Grande guerre, et qu'étudie ici même Anne Rasmussen. Or cette double adresse (Siess et Valency, 2002), en temps de conflit, n'est pas sans conséquence. Grâce à la médiation d'un pair, le locuteur peut faire entendre sa parole à un peuple ennemi auprès duquel, sans cela, il n'aurait pu bénéficier d'aucune écoute. Cet interlocuteur privilégié est d'ailleurs aussi celui qui veille à la traduction et à la diffusion du texte dans les journaux de son pays. Mais il y a plus. L'interaction épistolaire avec un intellectuel ou un savant de la nation adverse permet de placer - du moins d'essayer de le faire - le discours sur le plan de l'échange. Elle interpelle un individu singulier avec qui l'épistolier entretient des rapports personnels, ou pour lequel il éprouve une estime partagée. Aussi permet-elle d'émettre le dissensus sur le fond de la relation forte qui unit deux êtres d'élite : tout en se tournant vers le grand public, c'est encore à «l'ami» allemand ou français que l'on s'adresse au milieu de la tourmente. Le dispositif d'énonciation propre au genre lui permet ainsi de remplir une fonction toute particulière.

Et cela d'autant plus qu'on peut se demander dans quelle mesure la lettre à un correspondant appartenant à la nation ennemie vise vraiment l'adversaire et l'opposant. Le risque est grand qu'elle se contente de parler, à travers lui, à un public de compatriotes déjà acquis à la (bonne) cause. Comment s'adresser

2. J'emploie ce terme pour les textes de Renan et de Strauss même si son usage n'est pas attesté en 1870, la première occurrence dans le $T L F$ datant de 1882 (Lettre ouverte de Louis Pasteur à Robert Koch). Il n'en reste pas moins que l'échange épistolaire relève bien du genre en question, qui existe déjà de fait avant de revêtir sa dénomination actuelle. 
simultanément aux Allemands et aux Français ? Pour y parvenir, la lettre ouverte doit miser sur des points d'accord différents, voire totalement opposés. L'entreprise est malaisée, et on peut se demander si les diverses missives manifestent un véritable souci de la mener à bien. La lettre ouverte à un membre de la nation ennemie est-elle une simple mise en scène à l'intention de ses concitoyens, ou une véritable quête de dialogue ? Avant de se pencher sur ces questions dans les lettres ouvertes de Renan et de Strauss, d'une part, de Rolland et de Hauptmann, d'autre part, il faut esquisser une brève description de ces deux cas de figures.

Dans le cas d'Ernest Renan et de David Friedrich Strauss, il y va de deux érudits férus de la culture du partenaire ${ }^{3}$ qui s'adressent réellement l'un à l'autre à travers la presse des deux pays. L'échange épistolaire se donne de leur propre aveu comme un moyen de maintenir le dialogue au plus fort des combats, et de débattre de leurs dissensions sur le bon droit de chacun des pays engagés dans la guerre et sur le problème de l'appartenance de l'Alsace-Lorraine, sans couper le lien précieux entre la France et l'Allemagne. La situation devient de plus en plus délicate, et le ton s'envenime progressivement, au fur et à mesure que la victoire de l'armée allemande et sa volonté d'annexer l'Alsace et une partie de la Lorraine se précisent. La première lettre de Strauss, répondant à une demande faite par Renan dans une missive privée, est rédigée le 12 aout 1870 et publiée dans La Gazette d'Augsbourg le 18 du même mois; traduite et reproduite le 15 septembre dans Le Journal des débats, elle est suivie de la lettre ouverte de Renan rédigée le 13 septembre et publiée le 16 dans la même revue. Strauss réplique le 2 octobre toujours dans La Gazette d'Augsbourg, qui refuse cependant de reproduire la lettre de son correspondant français (que Strauss traduira lui-même et fera paraitre plus tard dans une brochure intitulée Krieg und Friede). Renan ne peut réagir que tardivement à un article auquel il n'a pu avoir accès au moment du siège de Paris. Datée du 15 septembre 1871, sa deuxième lettre ne sera publiée qu'en novembre dans La réforme intellectuelle et morale.

Le second cas est celui de Romain Rolland où l'auteur de Jean-Christophe, bien connu pour sa compréhension profonde de l'Allemagne, s'adresse le 2 septembre 1914 à un illustre écrivain allemand, Gerhart Hauptmann, qui a protesté dans le Frankfurter Zeitung contre les accusations de barbarie lancées par les Alliés (27 août 1914) ${ }^{4}$. Rolland se fonde sur des valeurs largement humaines et sur celles de la civilisation européenne qui est leur partage à tous deux, pour lui demander de faire entendre la protestation de l'intellectuel contre les exactions

3. À ce propos, on consultera avec profit Digeon, 1959, p. 178-214.

4. Nous utilisons la traduction française parue dans Cheval, 1963, avec certaines modifications issues de notre vérification de l'original allemand. 
de l'armée allemande en Belgique. La lettre, donnée à lire à tous les peuples entrainés dans la tourmente, demande le maintien de la solidarité des intellectuels au-dessus de la mêlée. Cet appel vaudra à Romain Rolland une cinglante réplique de son correspondant, publiée dans le Frankfurter Zeitung du 12 septembre 1914, et dont il peut prendre connaissance à travers la traduction partielle du Corriere della Sera (Cheval, 1963, p. 298, note 2). À cette réponse font écho en Allemagne une série d'articles indignés, parmi lesquels on peut trouver d'autres lettres ouvertes, comme celle de Karl Wolkskehl parue à la suite de celle de Hauptmann dans le même journal.

\section{Fonctions de la lettre ouverte en période de conflit armé}

Au-delà de divergences flagrantes, on peut relever entre ces échanges épistolaires une similitude importante de situation et de visée. En effet, ces lettres sont le fait d'intellectuels qui revendiquent hautement leur appartenance à une nation et qui pourtant se proposent de penser l'évènement avec une certaine prise de distance. En 1914 comme en 1870, au moment même où les soldats des deux pays se battent et meurent pour leur patrie, des hommes de sciences et de lettres qui se veulent les représentants d'une nation singulière demandent à conserver un droit à l'échange. Pour eux le partage de la parole, qui est quête de la vérité, reste valide fût-ce entre ennemis nationaux. C'est bien ce que semble suggérer Strauss lorsqu'il note à l'adresse de Renan, qui le sollicitait dans le cadre de leur correspondance privée :

Vous trouverez peut-être étrange aussi que ces lignes vous parviennent par l'intermédiaire d'un journal [...]. J'estime [...] qu'il peut y avoir quelque utilité à ce que, dans cette crise, deux hommes appartenant aux deux nations rivales, indépendants l'un de l'autre et étrangers à tout esprit de parti, échangent leurs vues sans passion, mais en toute franchise, sur les causes et sur la portée de la lutte actuelle. (Renan, 1992, p. 18)

Semblable échange s'effectue entre intellectuels qui s'octroient une reconnaissance mutuelle et éprouvent l'un pour l'autre une estime susceptible de favoriser l'écoute. C'est un dialogue entre pairs, que soulignent les adresses des lettres de 1870 et 1871, «Monsieur et savant maître», (ibid., p. 119), et les compliments réciproques : «Votre beau livre Jean-Christophe restera toujours vivant, pour nous Allemands, aux côtés de Wilhelm Meister et de Henri le Vert», écrit Hauptmann à Rolland (Cheval, 1963, p. 298). Mais c'est aussi une tentative de maintenir le lien entre représentants éminents de pays considérés à l'époque comme les piliers de la civilisation européenne, c'est-à-dire de la civilisation tout court. Renan mentionne la «force intellectuelle» de l'Allemagne (1992, 
p. 121) et rappelle que quand il l'a connue à travers Goethe et Herder, il a cru «entrer dans un temple» (p. 119). Rolland déclare : «Je connais la grandeur intellectuelle et morale de votre puissante race» $(1916$, p. 5), et reconnait sa dette envers les «penseurs de la vieille Allemagne» (ibid.). Strauss, dans sa première missive à Renan, s'exerce à comparer les mérites relatifs de deux cultures supérieures :

[L'Allemagne] commença par ce qui avait créé [...] le droit de la France à la suprématie européenne. Elle produisit une littérature, et on vit paraître une élite de poètes et de penseurs qui n'ont certes pas à craindre la comparaison avec les classiques français... (Renan, 1992, p. 109)

Dans l'article qu'il publie en même temps que sa lettre ouverte, «La guerre entre la France et l'Allemagne», Renan parle du face à face des deux peuples en des termes que Romain Rolland ne cessera de reprendre pendant la Grande Guerre : «Deux grandes races sont en présence; toutes deux ont fait de grandes choses, toutes deux ont une grande tâche à remplir en commun » (Renan, 1992, p. 99). Pour les penseurs français et allemands, la prise de distance critique en ce moment de crise semble d'autant plus importante, qu'elle est chargée de sauvegarder les valeurs de la civilisation que représentent les pays en guerre. Explicitement ou implicitement, la lettre ouverte assume dès lors une fonction capitale qui en décuple la portée.

Il résulte des deux premiers points (la prise de distance critique de penseurs situés, et la défense des valeurs de la civilisation), que le logos se doit d'endiguer, au moins partiellement, les affects débridés. L'entreprise est périlleuse, et la mission quasi impossible - personne ne l'ignore. Renan écrit ainsi à Ritter, son ami qui est aussi le traducteur de Strauss : «On fait ce qu'on peut pour faire entendre un peu de raison quoiqu'on sache fort bien que, dans le fracas des passions déchainées, elle ne peut guère être écoutée» (Psichari, 1947, p. 52). Le patriotisme exalté, dans ce cadre, n'est pas de mise. Dans sa lettre du 12 aout 1870 , Strauss entérine les vues de son correspondant sur le caractère nocif d'un sentiment national outrancier :

Certes, vous avez raison [...] quand vous déplorez qu'au lieu de l'entente entre les deux peuples, si nécessaire à l'œuvre de la civilisation, la haine, l'injustice et les appréciations passionnées soient pour longtemps à l'ordre du jour; vous avez raison encore quand vous déclarez que tous les amis de la vérité et de la justice doivent, sans oublier ce que leur imposent les devoirs patriotiques, se préserver de ce patriotisme partial qui rétrécit le cœur et qui fausse le jugement. (Renan, 1992, p. 108)

Publier une lettre ouverte à l'ennemi au plus fort des combats, c'est se garder d'une haine sectaire qui divise irrémédiablement les hommes; c'est tenter de protéger l'intégrité du jugement au milieu de la tourmente. En cette ère où 
les nationalismes se mettent en place, la lettre ouverte tente d'empêcher l'enrôlement des intellectuels dans les luttes du moment. En termes d'argumentation, on voit donc que les intellectuels, en s'engageant dans un échange épistolaire à la fois particulier et public, travaillent avant tout à relayer la violence par la parole raisonnée et à maintenir un lien entre ennemis. Il leur incombe de ramener les décisions capitales dans l'espace du débat public pour ne pas les abandonner à l'arbitraire de la force armée. Mais il y a plus. Il faut que la guerre reste une pratique réglée qui comporte ses limites. Même si les avis divergent sur ce point, il apparait que la force ne doit pas outrepasser certaines frontières si elle ne veut pas saper les fondements de la civilisation. Aussi les lettres ouvertes se donnent-elles pour tâche de débattre du bon usage de la victoire, de la légitimité de l'annexion des territoires (Renan et Strauss), des bornes assignées à la violence en situation d'invasion et d'occupation de territoires (Rolland et Hauptmann).

Cependant les lettres ouvertes remplissent une fonction supplémentaire, au sein de laquelle la relève des combats par la parole participe d'un mode différent. Elles analysent en effet l'évènement à partir d'une mise en opposition et en concurrence des identités nationales. Il en résulte que les deux épistoliers, non contents d'être les représentants de l'Allemagne et de la France, se proposent aussi d'échanger leurs vues, nécessairement divergentes, sur la nature de ces deux peuples (ou comme on disait communément à l'époque de ces deux «races»). Les lettres ouvertes deviennent un espace où les identités se négocient et se (re)définissent.

Elles constituent dès lors un terrain sur lequel les adversaires se mesurent en dehors du champ de bataille. Une lutte s'engage sur le plan des réputations : chaque nation veut projeter une image de soi aussi avantageuse que possible. C'est un peu l'équivalent de ce que l'on appelle aujourd'hui la guerre des médias : il ne s'agit pas seulement de savoir qui a gagné de fait, mais aussi qui a le beau rôle, c'est-à-dire qui a pour lui le droit. La question de la supériorité morale est un enjeu qui ne le cède pas en importance à celle de la suprématie militaire. Le genre de la lettre ouverte se prête particulièrement à ces visées dans la mesure où il permet d'esquisser derrière le destinataire direct, mais aussi derrière le public national, un auditoire élargi aux dimensions du monde civilisé. Romain Rolland, en sommant Hauptmann de condamner les exactions de l'armée en Belgique, ajoute que sa réponse est attendue par «l'opinion européenne» (1916, p. 8) et demande, si l'écrivain allemand approuve ces atrocités, que «l'opinion du monde [1']écrase» (ibid.). 


\section{L'ethos de l'intellectuel dans la tourmente : variations socio-historiques}

C'est dans cette perspective qu'il convient de confronter les échanges épistolaires de 1870 et de 1914 . Une première différence se dégage au niveau de l'ethos du locuteur (Amossy (éd.), 1999). Sans doute les épistoliers se présentent-ils toujours comme des intermédiaires, des hommes capables d'adopter une perspective large et de comprendre les deux parties. Mais qu'est-ce que l'impartialité dont ils se targuent ? Pour Renan et Strauss, c'est avant tout la capacité à exercer un jugement qu'aucune passion excessive ne fait pencher d'un côté de la balance. En s'adressant à son correspondant allemand, Renan évoque leur «libre et large sérénité» (1992, p. 120), et remercie Strauss de ses «hautes et philosophiques paroles» (p. 119). Strauss, qui effectue dans sa missive une analyse détaillée de la situation politique fondée sur une histoire raisonnée de l'Allemagne, justifie cette démarche en déclarant qu'à cause de la complexité d'une situation que les étrangers saisissent mal, il était nécessaire de «reprendre les choses de haut» (p. 118). Cette parole désengagée et réfléchie sert d'antidote à la violence guerrière : "Vos hautes et philosophiques paroles nous sont arrivées à travers ce déchainement de l'enfer, comme un message de paix » (p. 119), assure Renan. Selon l'idéal que les deux correspondants s'efforcent de maintenir, et que ne manquent pas d'infléchir les développements de 1871 (le siège de Paris, le projet d'annexion des provinces de l'Est), c'est donc l'image du lettré et du savant gardant ses distances qui apparait comme positive.

Pour Romain Rolland, l'impartialité se définit comme la position de celui qui ne fait pas pencher la balance pour l'un des deux partis. Aussi se décrit-il à l'intention de Hauptmann et des lecteurs allemands en médiateur qui fait jouer ses liens avec chaque pays en faveur d'un rapprochement : «J'ai travaillé, toute ma vie, à rapprocher les esprits des deux nations» (1916, p. 5). C'est précisément cet ethos de penseur impartial qui comprend et apprécie les deux nations aux prises que refuse Hauptmann lorsque, dans sa réplique cinglante à Rolland, il le renvoie à son image de Français qui ne peut voir la situation que d'un point de vue partial : «Maintenant que la sanglante coupure a détruit, comme tant d'autres, votre belle conception de la paix, vous regardez notre pays avec des yeux de Français...».

Et en effet, dans le conflit qui oppose en été 1914 des ennemis nationaux, on ne peut plus imaginer un échange de compliments courtois entre deux hommes qui s'efforcent de garder leur sérénité. Dans la lettre ouverte publiée dans 
Le Journal de Genève le 2 septembre 1914 (et rédigée le 29 aout), et donnée à lire au public français et aux Alliés aussi bien qu'à Hauptmann et à ses compatriotes, ce n'est pas la scénographie de la conversation philosophique qui est mobilisée. C'est bien plutôt celle d'une collaboration d'intellectuels appelés à prendre position contre les débordements illégitimes de la violence armée. L'auteur de Jean-Christophe incite celui qui est, comme lui, un dépositaire du patrimoine culturel humain à joindre sa protestation à la sienne face à la sauvage destruction des trésors d'art et de science de Louvain. Aux penseurs capables d'analyser ensemble la crise se substitue ainsi «la petite armée européenne qui est la garde d'honneur de la civilisation » (Rolland, 1916, p. 7), à savoir le groupe des intellectuels engagés qui doit veiller à ce que les limites ne soient pas outrepassées, et à ce que les piliers de la civilisation (selon l'expression plus tardive de Rolland) ne soient pas ébranlés. Il est intéressant de voir que la première lettre ouverte d'un Français à un écrivain allemand en 1914 est un appel à militer ensemble au nom d'une mission partagée, plutôt qu'à développer une réflexion commune.

Ceci nous mène à une deuxième dimension de l'ethos, celle de l'homme passionnément engagé. Alors que la guerre franco-prussienne voit la publication d'un dialogue qui se veut libre de tout entrainement passionnel, celle de 1914 mobilise consciemment le pathos, mettant face à face deux littérateurs indignés. Renan et Strauss se veulent des hommes de gout appartenant à l'élite de la société. Le Français se permet sans doute de reprendre son correspondant allemand en lui reprochant de ne pas avoir respecté «les délicatesses du galant homme» (1992, p. 152). Cependant ce reproche même implique que son correspondant partage ses prémisses sur l'existence d'une courtoisie qui doit régler les rapports humains. Il n'en va plus de même dans la lettre de Romain Rolland, qui ne se présente pas comme un échange poli entre lettrés, mais comme un appel pathétique à condamner les violences commises en Belgique. L'épistolier est un homme qu'anime le sentiment le plus vif, un sentiment moral qui le pousse à prendre la plume et à exhorter son adversaire. La chaleur de l'argumentation doit susciter un mouvement aussi passionné de la part du destinataire : "C'en est trop ! L'indignation du monde se révolte [...]. Vous acharner contre vos victimes, contre ce petit peuple belge infortuné et innocent ! Quelle honte !» (Rolland, 1916, p. 6). Les interjections, le rythme, tout signale un orateur emporté par un sentiment violent qu'il prend d'ailleurs soin de nommer dans une assertion généralisante. Son correspondant rétorque sur le même ton, maniant l'exclamation ( «Sans doute nos armées héroïques vous inspirent-elles la terreur !») aussi bien que l'argument ad hominem : «L'épithète de «Huns» a été inventée par ceux qui ont été déçus dans leurs projets d'attentats criminels contre la vie d'un peuple sain et profondément valeureux...» (Cheval, 1963, p. 299 300). Des deux côtés 
le ton est vif et le discours emphatique, si bien que chacun des épistoliers projette une image de polémiqueur passionné plutôt que de philosophe détaché.

L'ethos des épistoliers correspond ainsi à l'image qu'ils se font des fonctions de l'intellectuel et du médiateur culturel en situation de crise. La posture qu'ils adoptent détermine la nature des sujets qu'il leur est donné d'aborder, et le plan sur lequel ils se placent. C'est parce que l'effort de se dissocier autant que possible du point de vue passionné du patriote engagé leur apparait comme louable en 1870, que Strauss et Renan peuvent penser les conditions qui, dans la guerre, permettent de préparer la paix. Même si une dissension subsiste à ce propos Strauss appelle à une situation qui empêchera les Français de se livrer une fois de plus à leur appétit de conquête, Renan prévient contre les méfaits durables que susciterait une annexion définitive de l'Alsace-Lorraine - tous deux se situent dans le même horizon de coexistence pacifique prochaine. "C'est que, une fois la paix conclue, nous aurons tant à faire chez nous !» s'écrie Strauss (Renan, 1992, p. 149).

Dans ce contexte, l'échange épistolaire Renan-Strauss apparait comme une tentative partagée de penser conjointement non seulement le conflit, mais aussi la relation de la France et de l'Allemagne dans leur évolution. Les deux érudits qui cherchent à maintenir un lien amical «au milieu du tumulte de la guerre» (p. 118) se confèrent un statut d'analyste qui leur permet de situer le débat sur le plan de l'interprétation de l'Histoire. Ils discutent en particulier du «développement de l'unité allemande» (p. 120), question sur laquelle s'établit un véritable accord, et des torts respectifs des deux pays. Renan tente plus particulièrement de souligner son impartialité en critiquant son pays aussi bien que celui de l'adversaire. Il en va tout autrement de la correspondance entre Rolland et Hauptmann, où il ne s'agit plus guère de tracer un historique ni de comprendre des évolutions complexes. Les deux hommes se placent exclusivement sur le plan éthique, et se soucient peu de répartir également les fautes. Sous couvert de valeurs universelles et de vérités générales, chacun se fait l'avocat de son propre pays. Rolland condamne les atrocités commises par les Allemands en Belgique, Hauptmann dément, et justifie ses compatriotes. Il n'est question que d'un présent brulant, et chacun porte sur l'évènement un regard qui ne semble pas exempt de préjugé. Dans le jeu des accusations mutuelles, le dialogue tourne à la polémique. L'épistolier en oublie de s'appuyer sur des points d'accord partagés. Se fondant sur une série de pétitions de principe, il courtcircuite toute possibilité d'échange et rompt la communication. L'argumentation destinée à l'autre se transforme dès lors en lutte verbale donnée en spectacle à des tiers. Il s'agit d'exploiter la facture de la lettre ouverte pour feindre de s'adresser à l'allocutaire alors qu'on tente en réalité à la fois de rallier son propre camp autour d'un étendard, et de se donner raison aux yeux du monde civilisé. 


\section{La négociation des identités}

En même temps, l'échange épistolaire apparait comme l'espace où se confrontent et se négocient deux identités instables (ou déstabilisées), chacun des correspondants renvoyant à l'autre une image des deux nations qui devient un enjeu crucial dans un débat pourtant centré sur la recherche de l'entente et de la reconnaissance mutuelle. C'est ainsi que Strauss tend aux Français un miroir grossissant qui met en évidence leurs défauts. Il leur reproche

la recherche de l'éclat et de la gloire, le désir de briller, non par le travail tranquille à l'intérieur, mais par d'aventureuses entreprises au-dehors, la prétention arrogante d'être à la tête de la civilisation, le penchant à prendre les autres nations en tutelle et à les exploiter. (Renan, 1992, p. 116)

C'est donc «la passion nationale pour l'éclat, la gloire et les conquêtes» (p. 117), qui caractérise le Français, et en fait un adversaire de la paix que l'Allemand a toujours désirée et respectée :

Il est en outre singulier, et cela signale un remarquable retournement des choses, qu'un Français vienne nous prêcher la paix. Un membre du peuple qui depuis des siècles a brandi le flambeau de la guerre en Europe, sermonner ainsi le voisin qui n'a jamais rien pu faire d'autre qu'éteindre les feux que le premier avait porté dans ses villes et mis à ses semences? (p. 147)

Ainsi, la lettre de Strauss renvoie à Renan l'image d'un peuple dont l'humeur belliqueuse rendait la guerre inévitable, reliant étroitement la question de l'identité française à celle de la responsabilité de la guerre. Qui plus est, il oppose les valeurs allemandes à la décadence française : «Un peuple qui veut se conserver ne doit pas au fil des jours laisser corrompre par le plaisir et la splendeur le noyau de ses mœurs ». Ces accusations, Renan prend soin de les démentir en se démarquant de l'image du Français héritée de l'époque napoléonienne, et en renvoyant dans un jeu spéculaire aux Allemands le reproche formulé par Strauss :

Il ne sert à rien de dire qu'il y a soixante et soixante-dix ans nous avons agi exactement de la même manière, qu'alors nous avons fait en Europe la guerre de pillage, de massacres et de conquêtes que nous reprochons aux Allemands de 1870. Ces méfaits du Premier Empire, nous les avons toujours blâmés; ils sont l'œuvre d'une génération avec laquelle nous avons peu de choses en commun et dont la gloire n'est plus la nôtre. (p. 154)

Renan projette une image du Français qui puise à la fois à la "vieille école», et à ce que la France représente idéalement (en rupture avec la représentation périmée de l'Empire). La vieille école prône les valeurs du tact et de la délicatesse qui passent avant la passion (p. 152); la France qu'il évoque est celle de 
la Révolution et des principes du droit : «Notre politique, c'est la politique du droit des nations; la vôtre, c'est la politique des races : nous croyons que la nôtre vaut mieux». Et d'ajouter : "Cette conscience des peuples, nous l'avons créée dans le monde par notre Révolution; nous l'avons donnée à ceux que nous avons combattus et souvent injustement combattus; elle est notre dogme» (p. 154). L'insistance sur le droit et la politique libérale que les Français ont octroyés au monde permet à la fois d'argüer contre l'annexion de l'AlsaceLorraine, et de renvoyer aux Allemands l'image d'un peuple entrainé par la passion et le fanatisme, qui s'enferme dans un patriotisme étroit. Cette guerre des images s'accentue au fur et à mesure que le ton monte et que le débat vire à la polémique - chacun des épistoliers descendant dans l'arène et se livrant à une compétition qui se masque souvent sous les dehors du conseil prodigué à l'autre. Renan demande aux Allemands d'abandonner la position de leur "génération ultra-patriotique» qui «en repoussant tout ce qui n'est pas germanique pur» s'enferme sur elle-même (p. 161), se drape dans son orgueil et risque d'enfreindre d'importants principes moraux. Strauss projette dans son historique une image de la France sous les auspices de l'orgueil et de la soif de pouvoir, avide de gloire, naturellement belliqueuse, jalouse de toute suprématie et corrompue dans ses valeurs. Chacun demande à l'autre de revenir au meilleur de lui-même, et donne ses propres valeurs en exemple à l'adversaire. C'est ainsi que la confrontation polémique des images, tout en servant une argumentation sur la guerre, la paix et l'annexion des territoires, constitue aussi une négociation des identités.

À ce point précis, cependant, se fait jour une différence de poids. En effet, en 1870 , l'identité française confrontée à l'émergence d'une identité allemande en gestation entraine une négociation des spécificités nationales à travers le jeu des différences. À un moment de crise où s'élabore une nouvelle image de l'Allemagne aux yeux des citoyens de la nouvelle nation aussi bien qu'aux yeux des Français vaincus et occupés par l'armée de Bismarck, une quête identitaire se noue à travers le dialogue épistolaire. Lorsque éclate la guerre de 1914, par contre, les identités sont stabilisées. Celui qui, plus de quarante ans après Renan, entend maintenir le dialogue franco-allemand ne participe pas à une interrogation sur les identités nationales. Il tente au contraire de placer celles-ci sous les auspices d'une identité occidentale et européenne globale. Face à une conflagration généralisée qui précipite les nations européennes les unes contre les autres, il s'agit pour lui de replacer la France et l'Allemagne dans un cadre plus large et de remettre au premier plan la conscience d'un patrimoine commun menacé de destruction.

C'est dans ce contexte que la lettre ouverte de Romain Rolland en 1914 se donne comme une injonction aux membres d'une communauté fatalement 
divisée. Là où les savants de 1870 exploraient les différences, il accuse les similitudes; là où ils participaient d'une réflexion historique et philosophique, il se place sur le plan des valeurs partagées. On peut ainsi voir, une fois de plus, comment l'échange entre Strauss et Renan comporte une discussion de fond qui se maintient jusque dans la dissension, tandis que dans l'échange entre Romain Rolland et Gerhart Hauptmann, rien n'est vraiment débattu : un appel aux valeurs communes se heurte à une fin de non-recevoir argumentée avec force. On est passé d'une discussion malaisée mais persistante, au face-à-face d'un discours et d'un contre-discours qui ne ménage aucune passerelle.

Et en effet, la guerre des images nationales prend en 1914 une allure très particulière. Romain Rolland se situe par rapport au discours de son temps en se distinguant de ceux qui accusent les Allemands de barbarie. Il renvoie tout d'abord aux Allemands une image flatteuse, celle de la "grandeur intellectuelle et morale de [leur] puissante race», et évoque, sous le parrainage de Goethe, les «penseurs de la vieille Allemagne» auxquels un intellectuel français doit tant. Ces prémisses partagées permettent d'exiger de Hauptmann la plus sévère condamnation des sévices commis contre la Belgique. Leur comportement les rend indignes du rang qui leur avait été reconnu, celui de gardiens de la civilisation. L'épistolier tempère la violence de ses accusations en soulignant qu'il les adresse directement à l'ami allemand, et non à des tiers : "Ce n'est pas à l'opinion du reste de l'univers que je m'adresse contre vous. C'est à vous-même, Hauptmann » (Rolland, 1916, p. 7). Il demande à l'intellectuel allemand de condamner l'Allemagne des Huns au nom de celle de Goethe, pour ne pas déroger à ce que l'Allemagne se doit à elle-même.

L'injonction du Français germanophile à un interlocuteur privilégié, «J'attends de vous une réponse, Hauptmann, une réponse qui soit un acte» (p. 8), se heurte de sa part à une fin de non-recevoir. L'écrivain allemand rejette l'image négative de son peuple et en réfute le bien-fondé tant au niveau des faits qu'au niveau des principes. Il remet en cause la conception d'une guerre soumise à un code moral, et réfute les hiérarchies de son correspondant (la suprématie des œuvres d'art). Non seulement il pose que l'image qu'on renvoie aux Allemands d'euxmêmes est fausse, et s'explique par la rage des faibles et des vaincus, mais encore il relativise l'importance de la guerre des réputations :

Il vaut mieux qu'ils nous appellent fils d'Attila, fassent sur nous le triple signe de la malédiction et restent en dehors de nos frontières, plutôt qu'ils ne viennent écrire une épitaphe attendrie sur la tombe des chers petits-enfants de Goethe. (p. 299)

Dès lors l'entreprise de persuasion de Rolland, fondée sur le choix à effectuer entre deux modèles, les Huns ou les descendants de Goethe, échoue lamentablement. Hauptmann récuse la perspective éthique à prétention universaliste de 
Rolland et lui oppose celle de deux nations en guerre où la question de la représentation de l'autre est elle aussi sujette à une polémique violente. La négociation des identités, encore possible en 1870-1871, se transforme ainsi dès l'orée de la Grande Guerre en conflit ouvert au sein duquel le dialogue des pairs revêt une violence qui ne peut mener qu'au choc des positions adverses. La virulence des réponses de Karl Wolskehl et des autres intellectuels allemands à Rolland dans le Frankfurter Zeitung 5 confirme éloquemment cette «brutalisation» qu'étudient ici même Anne Rasmusen et Galit Haddad.

\section{Argumentation et Histoire culturelle}

À partir de ces deux exemples restés célèbres, on voit donc selon quelles modalités, mais aussi dans quelles limites étroites, le dialogue avec un représentant de l'ennemi reste possible en temps de guerre. Il n'est guère aisé, pour reprendre la formule de Massis, de parler lorsque la France (ou l'Allemagne) se bat. Sans doute le discours adressé à l' " ami» de la nation adverse est-il autorisé par une situation d'énonciation commune aux deux époques. Un espace occidental qui encourage l'échange entre lettrés les pose en porte-parole de la civilisation, et donc de la réflexion analytique et des valeurs morales; au cœur des passions nationalistes auxquelles ils ne peuvent échapper entièrement, ils se doivent de réguler la guerre pour qu'elle ne dégénère pas en violence anarchique. Cependant on voit comment le débat vire rapidement à la polémique, et la réflexion commune à une guerre des images nationales dans laquelle s'évanouit toute possibilité d'échange serein. On voit aussi que, dans ce cadre, l'interaction entre Rolland et Hauptmann ne jouit pas de la même latitude que celle de Renan et de Strauss en 1870-1871. Une vision contrastée de la relation francoallemande et de l'image que se renvoient réciproquement les deux nations se dégage en effet des divergences entre les deux échanges épistolaires.

C'est en ce sens que les modalités argumentatives des lettres ouvertes à «l'ami» de l'autre bord ne peuvent être comprises en dehors de leur situation d'énonciation. L'argumentation dans le discours, qui refuse de séparer texte et

5. Il faudra attendre la correspondance entre les pacifistes français et allemands publiée dans la Neue Zürcher Zeitung en 1916 pour que les modalités du dialogue se modifient quelque peu (Lorrain, 1999, p. 147-153). Mais elle relève ici d'une reprise, certes problématique, de l'échange entre membres d'une même cause globale qui implique une autre dynamique que celle étudiée ici. 
contexte, est donc par définition ancrée dans l'Histoire. En même temps, il apparait que les formes du dialogue, les stratégies de la polémique, ou encore les images de soi et de l'autre qui se négocient dans l'interaction épistolaire, peuvent alimenter l'Histoire culturelle en général, et les études qui se penchent sur la culture de guerre en particulier.

\section{Bibliographie}

ADAM J.-M., 1998, «Les genres du discours épistolaire», J. Siess (éd.), La lettre entre réel et fiction, Paris, SEDES.

AMOSSY R. (éd.), 1999, Images de soi dans le discours. La construction de l'ethos, Lausanne, Delachaux et Niestlé.

— 2000, L'argumentation dans le discours. Discours politique, littérature d'idées, fiction, Paris, Nathan.

AUDOIN-ROUZEAU S. et BECKER A. (éd.), 1994, Vingtième siècle. Revue d'histoire, $\mathrm{n}^{\circ}$ 41, janvier-mars, La guerre de 1914-1918. Essai d'histoire culturelle.

CHEVAL R., 1963, Romain Rolland, l'Allemagne et la guerre, Paris, PUF.

DIGEON C., 1959, La crise allemande de la pensée française (1870-1914), Paris, PUF.

HAUPTMANN G., 1914, Frankfurter Zeitung, 27 aout.

LORRAIN S., 1999, Des pacifistes français et allemands pionniers de l'entente francoallemande, 1870-1925, Paris, L'Harmattan.

MASSIS H., 1915, Romain Rolland contre la France, Paris, Floury.

PERELMAN C. et OLBRECHTS-TYTECA L., 1970, Traité de l'argumentation. La nouvelle rhétorique, Bruxelles, Éditions de l'université de Bruxelles.

PROCHASSON, C. 1993, Les intellectuels, le socialisme et la guerre. 1900-1938, Paris, Seuil.

PROCHASSON C. et RASMUSSEN A., 1996, Au nom de la patrie. Les intellectuels et la Première Guerre mondiale (1910-1919), Paris, La Découverte.

PROST A. et WINTER J., 2004, Penser la Grande Guerre. Un essai d'historiographie, Paris, Seuil (Points Histoire).

PSICHARI H., 1947, Renan et la guerre de 70, Paris, Albin Michel.

RENAN E., 1992, Qu'est-ce qu'une nation? et autres essais politiques, textes choisis et présentés par Joël Roman, Paris, Éditions Pocket (Agora).

RIOUX J.-P. et SIRINELLI J.-F. (éd.), 1997, Pour une histoire culturelle, Paris, Seuil. ROLLAND R., 1916, Au-dessus de la mêlée, Paris, Ollendorf (45édition).

SIESS J. et VALENCY G. (éd.), 2002, La double adresse, Paris, L'Harmattan. 


\section{Résumé / Abstract / Compendio}

\section{Dialoguer au cœur du conflit? Lettres ouvertes franco-allemandes, 1870/1914}

Le dialogue et l'argumentation entre ennemis nationaux peuvent-ils prévaloir dans une situation de guerre ? Comment le genre de la lettre ouverte contribue-til à semblable entreprise ? Deux échanges de correspondances publiés dans la presse par des intellectuels sont étudiés : Renan et Strauss pendant la guerre de 1870, Rolland et Hauptmann pendant la Première Guerre mondiale. L'analyse montre les possibilités d'un genre visant à empêcher la dissolution du logos par les passions nationalistes, l'impossibilité finale de maintenir un véritable dialogue, enfin les différences entre les échanges épistolaires de la guerre franco-prussienne et ceux de 1914-1918, éclairant ainsi l'impact d'une culture de guerre sur la rhétorique de son époque.

Mots clés : argumentation, culture de guerre, dialogue, lettre ouverte.

\section{Keeping dialogue in the heat of the conflict? Franco-german open letters, 1870/1914}

Can dialogue and argumentation between national enemies prevail in a situation of war? How can the genre of the open letter contribute to this enterprise? Two exchanges of correspondance published in the papers between French and German intellectuals are analysed, first during the 1870 war, then during WWI. Through the open letters of Renan and Strauss, and of Rolland and Hauptmann, both the possibilities are showed of a genre meant to prevent the dissolution of logos in violence and nationalistic passions, and the final impossibility to keep a true dialogue. It also stresses the differences between the Franco-prussian and the Great War epistolary exchanges, thus throwing light on the impact of a given war culture on the rhetoric of its time.

Key words : argumentation, war culture, dialogue, open letter.

\section{¿ Dialogar en pleno conflicto ? El caso de las cartas abiertas} franco-alemanas, 1870-1914

¿ El diálogo y la argumentación entre enemigos nacionales pueden prevalecer dentro de una situación de guerra? ¿ Cómo el género de la carta abierta podría contribuír a semejante empresa? Dos intercambios de correspondencias publicados en la prensa por intelectuales estan aqui estudiados : el de Renan y de Strauss durante la guerra de 1870, el de Rolland y de Hauptmann durante la Primera Guerra mundial. El análisis muestra las capacidades de un género que trata de impedir la disolución del logos en las pasiones nacionalistas, la imposibilidad final de mantener un verdadero diálogo, y por ende, apunta las diferencias entre los intercambios epistolarios durante la guerra franco-prusiana y los de 1914-1918, mostrando pues el impacto de una cultura de guerra sobre la rétorica de su época.

Palabras claves : argumentación, cultura de guerra, diálogo, carta abierta. 